Association for Information Systems AIS Electronic Library (AISeL)

Wirtschaftsinformatik Proceedings 2001

Wirtschaftsinformatik

September 2001

\title{
To Monitor or Not to Monitor - The Role of Trusted Third Parties in Electronic Marketplaces
}

Mareike Schoop

Informatik V, RWTH Aachen, schoop@cs.rwth-aachen.de

Thomas List

Informatik V, RWTH Aachen, list@cs.rwth-aachen.de

Follow this and additional works at: http://aisel.aisnet.org/wi2001

\section{Recommended Citation}

Schoop, Mareike and List, Thomas, "To Monitor or Not to Monitor - The Role of Trusted Third Parties in Electronic Marketplaces" (2001). Wirtschaftsinformatik Proceedings 2001. 44.

http://aisel.aisnet.org/wi2001/44

This material is brought to you by the Wirtschaftsinformatik at AIS Electronic Library (AISeL). It has been accepted for inclusion in Wirtschaftsinformatik Proceedings 2001 by an authorized administrator of AIS Electronic Library (AISeL). For more information, please contact elibrary@aisnet.org. 
In: Buhl, Hans Ulrich, u.a. (Hg.) 2001. Information Age Economy; 5. Internationale Tagung Wirtschaftsinformatik 2001. Heidelberg: Physica-Verlag

ISBN: 3-7908-1427-X

(C) Physica-Verlag Heidelberg 2001 


\title{
To Monitor or Not to Monitor - The Role of Trusted Third Parties in Electronic Marketplaces
}

\author{
Mareike Schoop, Thomas List \\ Informatik V, RWTH Aachen
}

\begin{abstract}
Electronic marketplaces provide a forum for bringing together sellers and buyers of certain goods or services. Today, a number of commercial marketplaces are in operation. To make a marketplace attractive to potential participants, it must be distinct from its competitors. One way to do so is to provide efficient functionalities complementing the existing support of search, negotiation, and fulfilment. In this paper, the emphasis is on monitoring and tracing functionalities that a marketplace can offer through a trusted third party (TTP) acting as a mediator. The possibility to trace back the reasons for a contract enables the participants to view the history behind an agreement. Monitoring certain phases of the business transaction can prevent a number of problems that can occur in electronic commerce. In this paper we will present different models of TTPs based on the monitoring and tracing facilities and illustrate them using a real-world scenario.
\end{abstract}

Keywords: Electronic marketplace, monitoring, traceability, trusted third parties

\section{Introduction}

Electronic markets have gone a long way from the first ideas [MYB87] to now. We have observed the shift from a research vision to a commercially exploited implementation of the vision. Nowadays, a number of marketplaces offer their services to the interested customer. While the first - and up to now most widely used - marketplaces are business-to-consumer (BtC) oriented, research focuses more and more on business-to-business (BtB) marketplaces. Here the biggest advantage over conventional commerce can be expected because electronic media such as the Internet help to reduce costs through many stages of the business process.

The most significant advances can be seen for large companies that reduce costs by coupling their IT structure through electronic data interchange (EDI) systems. However, the effort of gaining these profits is very expensive, especially because 
of necessary integration tasks. Usually only business partners with a sufficiently high business volume will cooperate using such systems.

Small and medium-sized enterprises (SMEs) demand different approaches for successfully utilising the Internet for business-to-business e-commerce (BtB EC). Here flexible systems are required that adapt themselves to the specific situations of the participating companies. One of the main advantages of the Internet for SMEs in addition to the ease of electronic communication is to find new business partners. Therefore, we argue that to support SMEs, marketplaces should cover in particular the early phases of a BtB process, namely the search and negotiation phase. A marketplace supporting these phases can then offer other features in addition to the support of these phases. One of the biggest problems for marketplaces is to be accepted by their customers who have to trust the marketplace in a certain way. In conventional commerce this trust was mainly built up through personal contacts with the business partners or intermediaries. In electronic commerce, different trust building measures have been discussed (e.g. [CDK00, ClLe99, MDS95, MLM94]). We will introduce a trusted third party (TTP) to the marketplace that has the role of a monitor being able to trace the business transactions, thereby, on the one hand, enhancing the trust of customers in the marketplace and, on the other hand, providing additional services that can make a marketplace more attractive to customers.

In the following section we will present our holistic view on the BtB process for SMEs. Section 3 contains a scenario of a business transaction in an electronic marketplace. In Section 4 we will present different models for tracing and monitoring electronic commerce; the scenario will be used to illustrate the applications of some of the models (section 5). Section 6 will then conclude the paper with a final discussion of the presented approach.

\section{A Holistic View on Electronic Commerce Processes}

The context of our work is an electronic marketplace that supports BtB EC. In this section, we will present a holistic view on an EC process. As will become clear later, such holistic view is particularly suitable for SMEs that are characterised by small or medium business volumes, high flexibility, and a large number of business contacts.

A business interaction starts with a search for new business partners. Search mechanisms should support intelligent search, e.g. to find related information such as similar products or companies providing services related to the products. Semantic networks can enable such search functionalities, e.g. by providing a powerful network of products that have links to related products or companies [LePa99] or by providing mechanisms for finding products not only by their exact name as 
in keyword search. The intelligent integration of product and business profiles enables the link of products and companies selling such products [QuSc00].

The buyer selects a number of companies as potential business partners and starts parallel negotiations. These interactions are characterised by a certain level of informality. The companies exchange information and make informal (i.e. nonbinding) enquiries. At one point, there will be a switch to a more formal form of interaction. The buyer decides to enter into serious negotiations with one company aimed at finalising a business deal. Negotiation support is envisioned here as an efficient message exchange that is based on speech act elements [Sea69, Hab85]. We will briefly describe the essential elements to pave the way for the discussion of traceability and monitoring in section 4; a more detailed discussion can be found in [ScQu01]. We aim for an electronic message exchange that enables the partners to express themselves in the best possible way while ensuring that the messages are unambiguous. Thus, it should be obvious that we do not argue for simple Email exchanges but rather for a semantically enriched form of electronic message exchange.

A supplier needs to know whether a message concerning certain goods (s)he receives is meant as an order or as a mere inquiry without obligations. To ensure unambiguity, the message type needs, therefore, to be specified. In our approach each message has got a content (describing what the message is about) and a message type (representing the way the message is meant, e.g. as a request, offer, counter-offer, assertion). To ensure unambiguity of the message content, a formalisation of the message content needs to take place. In general, the content of an utterance is often the source of many misunderstandings [Sch98]. On the one hand, the content of messages needs to be specified to enable queries such as "Which goods do we need to deliver to company A?" or "What did company B offer?" that concern the propositional content. On the other hand, systems that only offer predefined message contents appear too rigid and inflexible. Therefore, we take the message content as a semi-structured element. One possibility is to use XML representations of data. An example in the context of electronic negotiations is [Str01]. Messages can be classified into five classes [Sea69], namely assertive, commissive, directive, expressive, and declarative utterances. A trade-oriented set of subcategories of these five classes has been developed in order to be able to make statements about forces specific to that application area [Sch99]. Directive and commissive messages (e.g. requests and promises respectively) issue commitments for the recipient and the sender respectively. For example, a request made by a buyer and accepted by a seller implies that the seller is obliged to perform the action indicated in the message. In our approach, it is specified what kind of obligations the companies have already accepted during a negotiation. To ensure that all business partners know their duties, the obligations resulting from the exchanges need to be made explicit. An important requirement of electronic negotiations is that the exchanges should be logged to enable later access and traceability. Being able to trace messages of a particular negotiation process provides 
many advantages, e.g. enabling the evaluation of different negotiation strategies, providing a reminder of what has already taken place in a negotiation process, telling other parties involved about important information, providing the basis for monitoring mechanisms in case of later conflicts etc. The structured message exchange thus provides the basis for such monitoring and traceability (cf. section 4).

Once the negotiations are successfully completed, the next phase of interaction is entered, i.e. the fulfilment phase. Here both parties have certain obligations to fulfil. The payment needs to be managed and the goods will have to be transferred to the new owner. The IT support for the fulfilment phase highly depends on the business model the marketplace uses and on which additional information is available to the marketplace. For example, payment and delivery can be done via the marketplace - this is especially useful for goods such as software products where delivery can be done using the Internet. Even without such direct support, it is useful to inform the marketplace about the current status of the fulfilment phase. This could be used to visualise the status of the transaction for the two business partners or to find problems or errors in the fulfilment phase. In this work we will concentrate on the latter aspect.

It is important to mention that the sequence of an EC process as introduced here (search - negotiate - fulfil) does not necessarily need to occur in a strictly sequential order. For example, after unsuccessfully negotiating with a number of companies, the initiator might decide to go back to the search results and select different companies; after having drawn up a contract, the business partners might decide to re-negotiate, e.g. if the seller offers a product with better features. Furthermore, the sequence does not need to occur as a whole. For example, it is possible to skip the phase of searching if the potential business partners are already known. It should be obvious by now why the holistic view on BtB EC in electronic marketplaces is particularly suitable for SMEs. SMEs have a smaller business volume and are more flexible in their business relations than large companies. SMEs usually have a large number of business contacts reflecting their different needs in certain contexts. It has been observed that SMEs look for viable alternatives to the straight jacket of EDIFACT, which, for example, does not allow for negotiations, does not reflect the flexibility in the business interactions of SMEs, and can be expensive to implement and operate (cf. [KaWh97]). Rather SMEs look for less expensive systems that are adaptable solutions fitting the real needs of SMEs. 


\section{A Scenario}

In this section we will present a scenario ${ }^{1}$ illustrating a business transaction on a marketplace based on our approach as described in the previous section. The reader should bear in mind that negotiations can of course be more complex. However, our example is realistic enough for the present purpose.

Let us consider a small window building company called "Windows 2001 ". Its job is to provide windows consisting of metal frames and glass panes, tailored to the customers' demands. Customers of "Windows2001" are usually architects or construction companies. "Windows2001" is a member of a marketplace that brings together suppliers of window frames and panes and their prospective customers. The goods offered on the market are often combined with services such as creating a pane using glass that has certain features, e.g. insulation, a special colour or pattern etc.

"Windows2001" has been chosen by an architect to build and fit the windows for the front of a café. Since the front will only consist of glass panes and metal frames, good heat insulation is required. Furthermore, the windows must have a certain noise reduction factor. As our company has detailed knowledge about glass insulation, "Windows2001" can specify the quality of the glass according to the customer's needs. The specifications for noise reduction are provided by the architect of the café.

"Windows2001" acts as a customer on the electronic marketplace to look for companies offering panes with the required features. The intelligent search mechanism provides a list of five companies that have specialised in glass with heat and noise insulation. A buyer of "Windows2001" starts parallel negotiations with each of them. At one point, the buyer decides to start serious negotiations with one of the potential business partners, i.e. a company called "See Through". During the negotiation the questions about insulation w.r.t. heat and noise are discussed in detail by the two parties as the seller of "See Through" thinks that the specifications for noise reduction are too high. The buyer insists on the noise specifications as given by the architect but agrees to reduce the specifications concerning heat insulation as these seem to be unrealistically high. The two partners then agree on the costs and the time of delivery. A final contract containing all of the terms is then drawn up.

We would like to thank Metallbau Quix, Herzogenrath, for their help in creating a realistic scenario. 


\section{Monitoring Facilities in Electronic Marketplaces}

One aim of commerce via the internet is to enable direct business contact between two parties without the need of any intermediaries, called "disintermediation". The idea behind this trend was to reduce costs that arise when dealing with intermediaries. Nowadays a non-existence of intermediaries is more and more seen as a problem as the trust building tasks of the (conventional) intermediaries in particular are missing in e-business. The trend therefore at the moment is to reintroduce intermediaries to the e-business models [ClLe99; ScMü99] - this is sometimes called "reintermediation". In [CDK00] it is stated that intermediaries - from an economic point of view - lower the probability of unsuccessful trades, and consequently reduce the risk associated with trading.

The most important services an intermediary can offer are security services such as warranty of data integrity, anonymity, and authentification of communication parties. In practice, public and private key encryption and digital signatures are used in electronic commerce. The task of the intermediary is then for example to manage the public keys needed in such systems. Here the intermediary is often called Certification Authority (CA) or Trusted Third Party (TTP).

Contrary to these related approaches, our emphasis is on a different role of the TTP (i.e. the mediator), namely that of a monitor. We argue in addition to data security, other services are required to build trust in electronic commerce, especially in the case of SMEs. We, therefore, introduce two new, interrelated services for TTPs on electronic marketplaces: tracing and monitoring electronic commerce. The motivation for these services is that a contract a contract can be violated, obligations can remain unfulfilled, payment can be late etc. Thus, even if the negotiations ended successfully, it does not mean that the commerce transaction is successful. A central monitoring institution can trace the information in the contract back to its sources, i.e. where the agreement on that particular issue can be found. To make the monitoring acceptable for the business partners, an independent institution is required acting as the objective observer which in our case is the TTP. We see tracing as the ability of a TTP to store (parts of) the interactions between two parties on an electronic market place over the three phases searching, negotiation and fulfilment and to access them later on in an efficient way. The goal here is not only to secure the result of a negotiation, namely the contract, but also the reasons for a contract and, as far as possible, its fulfilment. Monitoring is thus the ability to recognise conflicts and errors in the e-commerce process between two parties and to react on these conflicts to help finding an agreement between the partners.

We already assumed monitoring to be a service of a TTP, not a service of the marketplace itself. The data that has to be collected to enable such a service provides a large amount of information about both partners and the marketplace itself. This information as a whole could easily be misused, e.g. to assess one of the companies. Thus the organisation having this information must be accepted by all 
participants of the marketplace and by the operator of the marketplace itself. Therefore, we do not see the marketplace We, therefore, do not see the marketplace provider but a trusted third party (TTP) as the provider for the monitoring services. Furthermore, to avoid an almighty TTP, there can be a network of several TTPs combined to provide the monitoring service such that none of the TTPs alone can analyse the data.

A related approach is that of Bolero.net [www.bolero.net], a standardised international platform for the exchange of trade documents. The main focus here is on security services that guarantee that documents are delivered securely and do arrive at their destinations. As bolero.net acts as an independent and trusted third parties, requirements regarding trust are similar to those for our approach.

The task of monitoring the electronic commerce process can be described by three dimensions: the phase to be monitored, the errors/conflicts to be recognised, the level of system activity (i.e. whether the monitor is active or passive). For a specific marketplace, the participants should define the monitoring role of the TTP by specifying the above dimensions. The following table shows the possible combinations of monitors - we will use the abbreviations later on in the text.

\begin{tabular}{|l|c|c|c|}
\hline \multirow{2}{*}{ MONITOR } & \multicolumn{3}{|c|}{ PHASES } \\
\cline { 2 - 4 } & Search & Negotiation & Fulfilment \\
\hline \hline Active & AS & AN & AF \\
\hline Passive & PS & PN & PF \\
\hline
\end{tabular}

Table 1: Combination of two monitoring dimensions

\subsection{Which Phase Is Monitored?}

The tracing and monitoring facilities are used to resolve problems between two parties on a marketplace. To do so, transaction data is stored and analysed by the TTP offering the service. Depending on the problems that should be resolved or assessed, the TTP does not need to capture all possible data but only stores a certain subset of the possible information. The first thing to look at to determine this subset are the different phases of the EC process. If the marketplace is only interested in errors of the fulfilment phase (wrong delivery, late payment etc.), there is no need to capture the search and negotiation phase. This distinction can also be more fine grained -some data can be stored while other data is not considered within the same phase.

Therefore, the data that has to be traced depends on what is necessary to resolve or assess a certain conflict that may happen during or after the e-commerce process and on what data should not be stored according to the stakeholders' requirements. 
The stakeholders should be able to force the marketplace not to trace certain information. This will lead to a reduced ability to recognise errors and to reconstruct negotiation and fulfilment situations. However, it is very important that the users remain the owners of their data and in this sense controls the TTP (and not vice versa). During the negotiation of a transaction, the participants should decide on the data they want to be monitored. It must be clear to both parties which implications the selection of traceable data has, in other words: it must be clear which conflicts can be recognised and what parts of the transaction can be reconstructed later on.

\subsection{Which Conflicts Should Be Recognised?}

The idea of a monitor is to detect errors or conflicts in the EC process. However, not every possible error that can be found by monitoring the transactions on a marketplace should be considered by the TTP. The question what conflicts or errors should or could be monitored strongly depends on what information is available on the marketplace. If the marketplace is not informed about the progress of the fulfilment, conflicts related to the fulfilment cannot be monitored. On the other hand, if the marketplace has a business model including payment and delivery through the marketplace, a better monitoring is possible. It is even possible for a company to allow the TTP to access the company's local ERP system. This enables the search for conflicts and inconsistencies such as: "You have offered to deliver 10000 pencils, but you only have 500 in stock".

Possible problems to detect include temporal issues (e.g. late delivery/payment), deadlocks in the negotiation process (company A waits for an event B1 of company B before being able to carry out event A2 while B1 depends on A2), satisfiability of obligations (fulfilling this obligation is impossible in the current context), priorities in negotiations (negotiations concerning a budget of $10000 €$ have a higher priority than the present process). In general, the conflicts can be divided into warnings that are only of interest to one party (e.g. capacity problems), warnings that are of interest to both parties (e.g. deadlocks), and errors/conflicts such as "payment did not arrive in time".

\subsection{Active and Passive Monitors}

There are two extreme cases concerning the level of activity. A passive monitor is mainly a data container and is realised as a pull-system, i.e. the monitor is only activated by the stakeholders of the marketplace. The TTP has to ensure that on demand every trace can be reconstructed in the specified way. If in the case of a violation of the contract the parties want to review the contract or the negotiations that led to the contract, the TTP will deliver that information. The other extreme case of monitoring is the completely active monitoring. Here the monitor is realised as a push system, i.e. the TTP constantly monitors every event that occurs on 
the marketplace (or in the transaction between the two stakeholders). This monitoring is strongly event-driven. The events can be distinguished into user-driven events, such as a stakeholder orders a certain good or service, and time-driven events, e.g. a payment or delivery has to be done until a certain date.

Monitoring of business transaction on a marketplace will generally be done in the fulfilment phase according to the contract that was drawn up in the negotiation phase. Conflicts that are monitored in this phase will usually consist of events that should have been fired before a certain date or time point. That date could be absolute or dependent on other events (e.g. the payment has to be done until 30 days after delivery). In contrast, the monitoring could start earlier, i.e. during the negotiation phase. The time of monitoring depends very much on what information is traced and/or monitored. In most cases, having a passive monitoring on the negotiation phase and an active monitoring on fulfilment will be a good start. A passive or partly active monitoring could even be useful for the searching phase as traces could be used to assess the negotiations or to help the stakeholder when negotiations with one party failed and the search is restarted.

Active and passive monitoring can also be combined in other ways. There could be an active monitoring on the negotiation phase for only one stakeholder - who is interested in as much information as possible - and a passive monitoring for the other stakeholder. Another alternative is that the TTP could actively monitor only some conflicts and just trace the rest of the transaction using passive monitoring. We will describe certain combinations in the following section.

\subsection{Data Model for Tracing the Negotiation Phase}

Monitoring the fulfilment phase is sometimes implemented in existing ERP-systems. The monitoring mainly consists of checking whether delivery and payment is on time. Monitoring the negotiation phase is a more difficult matter and thus requires more sophisticated mechanisms since the emphasis here is on inter-business processes rather than on intra-business processes that are the main focus of ERP systems. Monitoring includes testing the satisfiability of obligations, preventing deadlocks, checking the current context of interaction w.r.t. other negotiation processes. In this section we will discuss the basis for such tracing features.

To enable traceability on the negotiation phase, negotiation is based on the model in figure 1 (cf. [ScQu01]). We distinguish between documents (e.g. contracts), messages that lead to documents, and stakeholders (partners) that contribute to a contract. Several messages and documents are bundled by a negotiation. Documents are versioned via the "is successor of" association and negotiations build hierarchies of sub-negotiations (the "is subneg of" association). Message is specialised into subclasses according to the trade-oriented illocutionary forces as described in section 2.2 . 


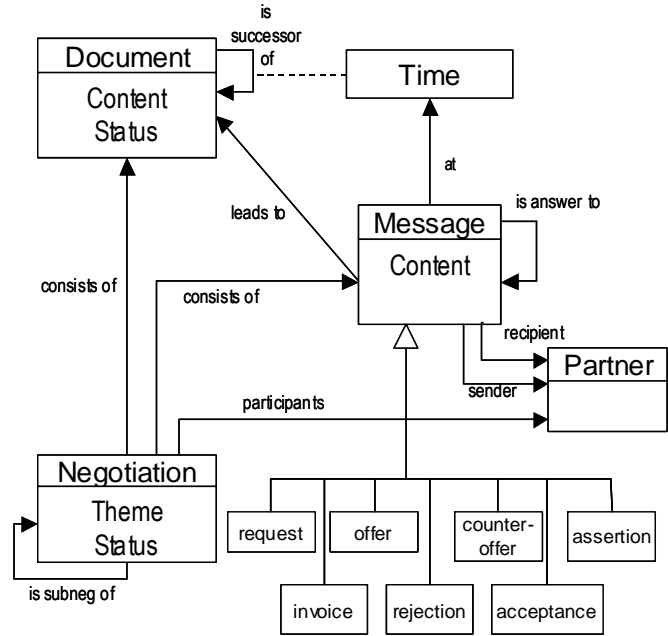

Fig. 1: Negotiation Model

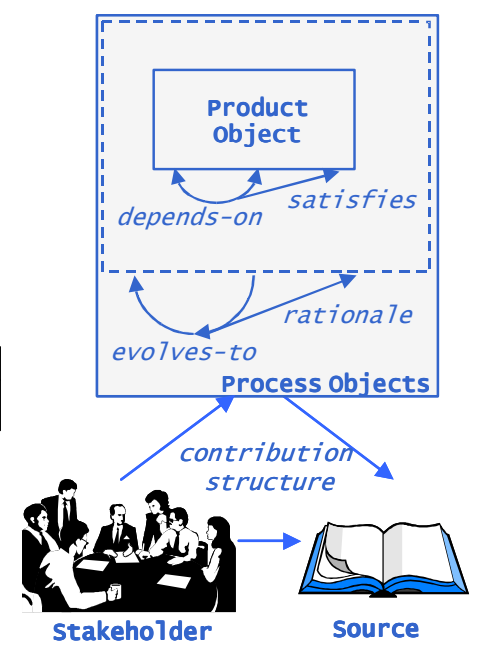

Fig. 2: Traceability Meta Model

The model shown in figure 1 is influenced by the traceability meta model shown in figure 2 [RaJa01]. Here different dimensions of traceability information are described: what information is represented (product objects in fig. 2, negotiations in fig. 1); who the stakeholders are that play different roles in the creation, maintenance and use of various objects (stakeholder in fig. 2, partner in fig. 1); how and where the information is represented (sources in fig. 2, documents in fig. 1); why a certain object is created, modified or evolved (contribution structure and rationale in fig. 2, message in fig. 1); when this information was captured, modified and evolved (time in fig. 1). The support of this meta model ensures traceability of the negotiations.

\section{Applications of the Models}

We will now look at some combinations of the three dimensions of monitoring introduced in sections 4.1-4.3. (see table 1). Three different models of a TTP are presented and illustrated using the scenario of section 3 .

\section{Example 1}

The first marketplace uses the following combination (PN\&AF).

Both the negotiation and fulfilment phases are traced. To enable the tracing of the fulfilment phase the marketplace is informed about external events such as payment or delivery by the stakeholders. Each external event needs to be approved by the second party. For example, if company A pays the stipulated amount it notifies the marketplace about it. The receiver of the money, company B, notifies the mar- 
ketplace once it receives the money. The marketplace monitors the fulfilment phase w.r.t. conflicts concerning time-points and deadlines. Warnings are created if one party has not yet fulfilled parts of the contract shortly before a deadline and error messages are issued if a deadline is missed. The monitoring is active for the fulfilment phase. As we trace the negotiation, we have passive monitoring on the negotiation phase.

The marketplace supports negotiations as described in section 2. The contract based on the electronic negotiation described in section 3 contains the following articles about the delivery of a window pane: the size of the pane, the colour of the pane, the specifications for heat insulation, the specifications for noise reduction, the price, the date of payment (a prepayment is payable within 10 days after the conclusion of the contract; the remaining amount needs to be paid 30 days after delivery), and the deadline of delivery.

Some of the reasons for the articles of the contract are sketched in section 3 . Shortly after the conclusion, "Windows2001" pays the prepayment according to the conditions. The marketplace is notified about this and the action is approved by "See Through". While producing the pane, "See Through" finds out that by slightly reducing some of the specifications concerning noise reduction the pane could be produced much cheaper. "Windows2001" is contacted and asked whether a modification of the contract is possible. "Windows2001" is not sure about the reasons of the specifications any more and uses the negotiation trace of the TTP to find out. The trace states that the specifications were done by the architect. "Windows2001" then contacts the architect and together they decide to change the specifications. Ten days before the delivery deadline, an event is fired by the TTP that checks whether the delivery has already been done. This trigger was generated from the contract articles. As this is not the case, a warning or notification is sent to "See Through" noting that delivery of the pane is due in ten days. This is no problem, the pane is ready for delivery. A few days later, "Windows2001" receives the pane and the marketplace is notified about the delivery. A new set of triggers is generated according to the agreement about payment. After the final payment the transaction is closed and the traces and contracts are transferred to the TTP's archive.

\section{Example 2}

The second marketplace uses the following combination (AN\&AF). The tracing of the negotiation and fulfilment phase is similar to the one describe in example 1. The marketplace monitors the negotiation for conflicts regarding prospective obligations, inconsistencies with existing obligations, and the possibility of fulfilling the obligations. The fulfilment phase is monitored similar to the first example. The monitoring is active for the negotiation and the fulfilment phases. Tracing of the negotiation and fulfilment phase as described in example 1. The marketplace monitors the negotiation for conflicts regarding prospective obligations, inconsis- 
tencies with existing obligations, and the possibility of fulfilling the obligations. The fulfilment phase is monitored similar to the first example.

"Windows2001" and "See Through" negotiate about 50 glass panes of the required specifications (size, colour, heat insulation, noise reduction). "See Through" offers to deliver the panes within a fortnight. Here, the active monitor interferes. There is a link between the monitor and the company's internal resource management. This could, for example, be the company's ERP system or a subset of data that the company has made available to the TTP. The TTP as an active monitor now issues a warning to the representative of "See Through" that there are not enough resources for the amount of panes with the required insulation factor. Therefore, it is not possible to deliver the panes within a fortnight. "See Through" first needs to order the required resources from the wholesaler. The consequence of this instance of active monitoring is that "See Through" modifies its original offer in that it now offers to deliver the panes within 20 days.

\section{Example 3}

Here the marketplace uses the following combination (PN). Only the negotiation phase is traced. There is no tracing of fulfilment, so the constructs of notification and approval are not required. There is no monitoring of conflicts. We have a passive monitoring role for negotiation so that the TTP acts as a pure archive for the negotiation process and the contract.

"Windows2001" and "See Through" start to negotiate about the delivery of the window panes as described in section 3. After complicated negotiations about the detailed conditions both parties come to a final agreement. In the final offer of "See Through" the noise insulation does not fit the specifications any more. "Windows2001" complains and uses the negotiation trace of the TTP to prove that there was an agreement on the correct specifications early during the negotiation.

\section{Conclusion}

In this paper we presented a holistic view on business-to-business electronic commerce transactions in a marketplace. Starting from a search for potential business partners, followed by electronic negotiations, a contract is drawn up with certain obligations that need to be fulfilled in the fulfilment phase. Trust plays a very important role in electronic transactions as has been noted by many researchers [ClLe99; MDS95]. One service that can help to build trust in electronic commerce is the monitoring and traceability function. To ensure that all partners involved acknowledge that certain data is monitored during a transaction, an independent organisation needs to act as a monitor. We have introduced the notion of a trusted third party (TTP) in that context. There are other approaches using second party control in which the parties place certain restrictions on each other. How- 
ever, in our experience an independent institution is more acceptable. Furthermore, the monitoring role can be combined with other services such as trust services, financial information etc. to enhance trust in a certain marketplace.

There can be different types of monitoring that a TTP can provide. In this paper, three dimensions have been introduced that can classify the roles of a TTP as a monitor in an electronic marketplace. The next step of our research will continue to implement the key models, some of which were introduced in this paper. Empirical studies will show in more detail for which context the different models are most appropriate. Furthermore, it will be assessed to which level companies will use monitoring services.

To monitor or not to monitor - this is no question to us. Monitoring is an additional feature of a marketplace that can offer valuable services for the stakeholders. The users decide beforehand what data they want a TTP to see, which data should not be accessible, and in general which model to apply. Thus, the users remain in control of their data and make the final decision of what to monitor. To monitor and what to monitor - that is the question.

\section{References}

[CDK00] A.M. Chircu, G.B. Davis and R.J. Kauffman: Trust, Expertise, and E-Commerce Intermediary Adoptions. In Proceedings of the 2000 Americas Conference on Information Systems, Long Beach, CA, 2000.

[ClLe99] T.H. Clark and H.G. Lee: Electronic Intermediaries: Trust Building and Market Differentiation. In Proceedings of the Thirty-Second Annual Hawaii International Conference on System Science, IEEE Computer Society, 1999.

[Hab85] J. Habermas: Theorie des kommunikativen Handelns. Vol. 1: Handlungsrationalität und gesellschaftliche Rationalisierung., Suhrkamp, 1985.

[KaWh97] Kalakota and A.B. Whinston: Readings in Electronic Commerce. AddisonWesley, 1997.

[LePa99] C.J. Leune and M.P. Papazoglou: Classification Mechanisms and semantic searches for trading-information. Deliverable 1.1, MEMO project, available at http://www.abnamro.com/memo, 1999.

[MDS95] R.C. Mayer, J.H. Davis and F.D. Schoormann: An Integrative Model of Organizational Trust. Academy of Management Review, 20(3):709-734, 1995.

[MLM94] M. Merz, W. Lamersdorf and K. Müller: Trusted Third-party Services in COSM. Electronic Markets, 4(2):7-8, 1994.

[MYB87] T.W. Malone, J. Yates and R.I. Benjamin Electronic Markets and Electronic Hierarchies. Communications of the ACM, 30(6):484-497, 1987. 
[QuSc00] C. Quix and M. Schoop: Facilitating Business-to-Business Electronic Commerce for Small and Medium-Sized Enterprises. In Proceedings of First Intl. Conference on Electronic Commerce and Web Technologies, EC-Web 2000, pp. 442-451, Springer Verlag, 2000.

[RaJa01] B. Ramesh and M. Jarke: Towards reference models of requirements traceability. IEEE Transactions on Software Engineering, 27(1):58-93, 2001.

[Sch98] M. Schoop: Towards Effective Multidisciplinary Communication: A LanguageAction Approach to Cooperative Documentation Systems. PhD Thesis, The University of Manchester. UK, 1998.

[Sch99] M. Schoop: A Theoretical Framework for Speech Act Based Negotiation in Electronic Commerce. In Proceedings of the Sixth Research Symposium on Emerging Electronic Markets (RSEEM), Arbeitsbericht Nr 72, Institut für Wirtschaftsinformatik, Universität Münster, pp. 79-89, 1999.

[ScMü99] D. Schoder and G. Müller: Disintermediation versus Intermediation auf elektronischen Märkten am Beispiel 'Trusted Third Parties'- Eine empirisch gestützte Einschätzung. In Tagungsband zur Wissenschaftlichen Jahrestagung 1999 des Verbandes der Hochschullehrer für Betriebswirtschaft e.V., Bamberg, 1999.

[ScQu00] M. Schoop and C. Quix: Towards Effective Negotiation Support in Electronic Marketplaces. Proceedings of the 10th Annual Workshop On Information Technologies and System, WITS 2K, Brisbane, Australia, pp. 1-6, 2000.

[ScQu01] M. Schoop and C. Quix: DOC.COM: Combining Document and Communication Management for Negotiation Support in Business-to-Business Electronic Commerce. Proceedings of the 34th Hawaii International Conference on System Sciences, Maui, Hawaii, 2001.

[Sea69] J.R. Searle: Speech Acts - An Essay in the Philosphy of Language. Cambridge University Press, Cambridge, 1969.

[Str00] M. Ströbel: Communication Design for Electronic Negotiations. To appear in: Proc. Of the 10 $0^{\text {th }}$ Intl. World Wide Web Conference (WWW10), Hong Kong, 2001. 